\title{
What Is Unique About Mechanochemical Reactions?
}

\author{
L. TAKACS* \\ Department of Physics, University of Maryland Baltimore County, Baltimore, MD 21250, USA
}

\begin{abstract}
Mechanochemical reactions can provide compounds, phases, and microstructures that are essentially different from the products of ordinary reactions. In this paper, the origin of this uniqueness is discussed in light of the recent advances of the field. It is claimed that the local availability of large batches of energy, well above $k T$, is the key feature of mechanochemical reactions. As a consequence, reactions that cannot occur thermally become possible, similarly to the reactions induced by the energy of photons in photochemistry. However, the situation is more complex, as macroscopic deformation affects many defect sites simultaneously. The direction of the mechanical load relative to the orientation of a molecule or the crystallographic axes of a solid can be important. Many mechanochemical reactions of organic compounds take place at low milling energy that is not sufficient to break primary bonds, but the gentle mechanical grinding can influence the relative position of macromolecules, leading to the formation of unique cocrystals and compounds. In inorganic systems, unusual products form due to forced mixing and the high defect density generated by intense milling.
\end{abstract}

DOI: 10.12693/APhysPolA.126.1040

PACS: 81.20.Ev, 82.33.Pt, 82.30.-b

\section{Introduction}

Mechanochemistry has been practiced for over a century, but the question of exactly which processes are the subject of mechanochemistry is still debated. Moreover, as new areas of science and technology discover the possibilities offered by mechanical treatment, the views on mechanochemistry shift to accommodate new systems and phenomena. Therefore, it is necessary to periodically reevaluate our fundamental notions of mechanochemistry. The most important development of the past several years was the increasing use of mechanical activation in organic chemistry, particularly in organic synthesis. While high-energy milling is used to break primary bonds, lower energy mechanical mixing/milling is used to facilitate solvent-free reactions and to prepare co-crystals. Mechanophores, are incorporated into materials to give them signaling or self-healing ability. A special volume of Chemical Society Reviews [1] and the recent Faraday Discussion on mechanochemistry [2] reflect many of the new developments.

Over most of this paper, a process will be considered "mechanochemical," if its initial step is the breakage of a primary bond, induced or promoted by mechanical force. This definition is similar to the one recommended by Kaupp [3], although it is used in a broader sense. For example, shattering a particle into pieces is usually considered a purely mechanical process. But it requires the mechanical breakage of bonds, thus it is mechanochemical at its core. Breaking bonds creates reactive surfaces and results in surface reconstruction and reactions with the surrounding medium. Mechanochemical reactions are not restricted to solids. Vigorous mixing or sonication of a liquid can also result in molecular forces that directly strain and break bonds.

\footnotetext{
* corresponding author; e-mail: takacs@umbc.edu
}

A broader definition was given by Baláž [4]: "Mechanochemistry is a branch of chemistry which is concerned with chemical and physico-chemical changes of substances of all states of agglomeration due to the influence of mechanical energy." According to this definition, any process that involves mechanical action beyond gentle mixing of a solution falls under the scope of mechanochemistry. Detailed analysis of individual cases show that mechanical action indeed plays some role in a variety of processes, thus taking a broad view has its merit.

It is possible that the initial step of a mechanochemical reaction is the emission of low-energy electrons rather than the breakage of a primary bond. The absorption of these electrons creates negatively charged radicals that can initiate further chemical changes. This mechanism will not be discussed here, but it is very important in tribochemical processes where sliding is the main form of mechanical action [5,6]. Obviously, sliding between particles also takes place during mechanochemical processing.

The rate of bond breaking can be described by an Arrhenius equation, modified with a term for the mechanical tension applied to the bond $[7,8]$ :

$$
k(T)=A \exp \left[-\left(E_{a}-\alpha F\right) /\left(k_{\mathrm{B}} T\right)\right] .
$$

Here $F$ is the external tensile force and $\alpha$ is an appropriate characteristic length. If $\alpha F$ is small, the bond breaks due to thermal excitation; the mechanical tension only lowers the activation energy. If $\alpha F$ is larger, the effect of the mechanical tension becomes dominant; the difference is more pronounced at low temperature. The bond brakes by the tension directly, if $\alpha F \geq E_{a}$. In most cases the mechanical and thermal effects are mixed.

In many practical processes mechanical action - usually ball milling - is used to induce reactions in the solid state at ambient temperature. Usually the products are similar to those obtained by conventional methods and mechanochemistry is used only to make the process 
"greener" or faster. But mechanochemical reactions can also be unique, and their products can be essentially different from the compounds and phases obtained by conventional chemistry. In most of those cases the reaction begins with the direct mechanical breaking of a primary bond. Much of this paper deals with such situations.

\section{A bit of historical background}

It is customary to consider M. Carey Lea the "father of mechanochemistry" based on his work at the end of the 19th century [9]. Chemical reactions had been induced by mechanical action earlier, but he was the first to show that mechanical action can initiate reactions that are distinctly different from the reactions induced by heat in the same system. His results on the decomposition of silver and mercury halides are still frequently cited, not only as historic milestones, but also as one of the clearest examples of the uniqueness of mechanochemical reactions [10].

Lea was keenly aware of the significance of his results. W. Spring studied chemical reactions induced by compression about a decade earlier [11] and raised a priority claim against him [12]. In his rebuttal, Lea stated that the ability to induce chemical reactions with mechanical means had been know for at least half a century, well before Spring's work. Thus his experiments were interesting, but not particularly new. As Lea wrote:

"My dispute with Prof. Spring derives from the fact, that the author does not discriminate between endothermic and exothermic reactions. In order to carry out an endothermic reaction, the driving energy must be provided during the entire duration of the reaction, not only to initiate it. Accordingly, if the reaction is induced by mechanical force, it requires the steady conversion from mechanical into chemical energy..." [13]. (Translated from German by the author.)

Although this assessment is technically incorrect as endothermic reactions do occur spontaneously using heat from the environment, Lea was right that obtaining products that are different from the products of heating was of fundamental novelty and importance.

For the next several decades, scientists investigating mechanochemical processes were interested in potential applications rather than fundamental questions about the relationship between mechanical deformation and chemical changes. It was only during the resurgence of mechanochemistry after 1960 , that basic questions about the nature of the process emerged again [9].

\section{Concentration of energy}

Lea's early interest was the chemistry of photography; he even published a book on photography in 1868 [14]. In 1966, he compared the effects of light and pressure on a photographic plate. When he turned to mechanochemistry, his choice of model materials - silver and mercury halides - reflected his background in photochemistry. We follow Lea's approach by beginning the discussion of mechanochemistry with a comparison to photochemistry.

In ordinary thermochemistry, the energy barrier against changing the bond structure is overcome by thermal fluctuations. A reaction takes place at a reasonable rate only if the activation energy is not more than a few times $k T$. Photochemical reactions can be different, as the energy delivered by a single photon, is many times $k T$, thus creating high-energy excited states - such as broken primary bonds - becomes possible.

The local availability of large batches of energy is a key feature also of mechanochemical reactions. But compared to photochemistry, there are added complication: While photochemical reactions take place one photon one reaction site at a time, mechanical action on a bulk sample involves a macroscopic amount of energy that gets distributed into a large number of active sites. The distribution is not even. For example, plastic deformation takes place at slip planes and the slip is mediated by dislocation motion. Only the dislocation cores experience high energy density. In brittle materials the tip of a fracture is the active, highly perturbed site. The energy is concentrated into contact points during sliding. The locally available energy substantially exceeds the energy of thermal fluctuations, thus reactions with large energy barrier become possible.

\section{Bond selection, anisotropy}

The simplest mechanochemical process, that involves a single site like in a photochemical reaction, is breaking a single-atom chain of atoms in an atomic force microscope (AFM.) This has been demonstrated for gold [15] and was also simulated using ab initio molecular dynamics [16]. The ultimate strength of the wire is $1.5-1.8 \mathrm{nN}$ according to both experiment and calculation. The calculations also reveal that the elongation of the bonds does not increase monotonously toward the middle of the chain of atoms. Some bonds get longer and weaker, but others become shorter and stronger. Which bond will eventually break is hard to predict without detailed analysis. Also, when a bond breaks, much elastic energy is stored in the neighboring bonds, resulting in intense vibrations, equivalent to very high local temperature. This is an important feature of any mechanochemical reaction.

If a more complicated molecule is attached to a surface then pulled away, one can also ask what kind of bond breaks. This question was studied in an early work by Beyer, who considered an amylose polysaccharide molecule anchored to a glass surface [17]. Pulling on this molecule stretches five different kinds of bonds - C-C, C-N, C-O, Si-C, Si-O - either in the spine of the molecule or at the glass surface. Calculations of the bond strength on small molecules with similar bonds revealed that breaking the $\mathrm{Si}-\mathrm{C}$ bond requires the smallest force, while the $\mathrm{Si}-\mathrm{C}, \mathrm{C}-\mathrm{N}$, and $\mathrm{C}-\mathrm{O}$ bonds have similarly low binding energies and would break upon heating with 
almost equal probability. Therefore, mechanical action and heat would have very different consequences.

Pulling away an ethylthiolate $\left(\mathrm{CH}_{3}-\mathrm{CH}_{2}-\mathrm{S}\right)$ molecule from a $\mathrm{Cu}$ surface provided further surprises [18]. Thermal motion weakens and eventually breaks the C-S bond. But under tension, the same bond hardens, $\mathrm{Cu}$ atoms are pulled out of the surface and eventually a $\mathrm{Cu}-\mathrm{Cu}$ bond breaks and one or two $\mathrm{Cu}$ atoms are removed from the surface. These and other examples are discussed in detail in a review by Ribas-Arino and Marx [19].

The molecules in the previous examples contained a single spine, thus the mechanical action could be described as simple tension. But molecules with more complex connectivity, containing multiple rings or cages, can be pulled at different points, at more than two points, or in different directions. Even if the external load is simple tension, the force can be distributed inside the molecule in a variety of ways. Which bonds will eventually break depends on the details of the mechanical load. The questions of where, how, and in what direction external forces are applied are also relevant to crystals of organic molecules.

AFM has been used to study individual macromolecules under tension extensively [20]. Another method is offered by polymer sono-mechanochemistry [21]. A mechanophore (stress sensitive molecule) is dissolved in an appropriate solvent. Then two long polymer chains are attached to the desired points of the molecule, using the methods of polymer chemistry. In this way, a long molecule is obtained with the unit of interest in the middle. Ultrasonic cavitation can be used to tug on the polymer chains and exert tension on the mechanophore. Changes of the properties, such as luminescence or scission of the molecule are observed.

Is there any direction dependent behavior in the mechanochemistry of inorganic materials? There must be, as the elastic and the plastic properties are direction dependent, thus the chemical effects of mechanical load must be direction dependent also. But the materials used in practice are usually polycrystalline and the direction-dependent effects are probably small. Nevertheless, fundamental understanding of the mechanism of mechanochemical processes requires knowledge of the processes on the atomic and nano scales where the properties depend on crystallographic directions.

Ideally, direction-dependent effects should be studied in solid-solid reactions between two single crystals under controlled mechanical deformation. It is easier to study processes between the surface of a single crystal and a liquid. The dissolution around a hole in a single crystal K-alum sample under stress showed clear direction dependence [22]. The stress corrosion of single crystal surfaces with external load in different directions could be studied. The particle emission of cleaved crystals was investigated some time ago [23]. It would be interesting to see, whether cleaving a crystal along different lattice planes would result in the emission of different species. Investigating the early stages of thermal decomposition or phase transformation of single crystals under stress could provide interesting results. In general, investigating direction-dependent mechanochemical effects could provide many interesting results.

\section{Materials that cannot form in any other way}

Mechanochemistry is a practical subject; its goal is not only to understand the mechanism of natural maninduced processes, but also to produce useful materials. Organic mechanochemistry offers many possibilities, although most of them do not involve the direct mechanical manipulation of primary bonds. In some cases, the problem is the lack of solubility. For example, fullerenes do not dissolve in ordinary solvents, but chemical reactions can be induced under milling [24]. Even if the reactants can be dissolved easily, the reactions can be faster or more selective upon mechanical milling. This is the case for some metal-organic complexes [25]. The mechanochemical preparation of cocrystals [26] has important application in pharmaceutical chemistry. Eliminating or minimizing the use of solvents has obvious implications from the point of view of green chemistry. In some cases a small amount of solvent is essential to carry out the reaction or to modify its outcome [27]. Some reactions are essentially thermochemical, milling is needed only to bring together the reactants in appropriate relative configurations [28].

Is it possible that mechanical mixing-milling results in organic molecules that cannot be obtained in any other way? Most probably, yes, but the key is not breaking covalent bonds at unusual places. More likely the grinding action will be quite gentle, used only to bring, in fact, force, together reactants that do not mix otherwise, or, if mixed in a solution, do not come together in the configuration that is required for a certain reaction. Once the required groups come into proximity, a click reaction takes place and the unique molecule forms [29].

Mechanochemistry is not restricted to the solid state. Shear forces in a liquid can be strong enough to affect substantial changes. Sono-mechanochemistry of polymers was discussed earlier. A very recent example is the preparation of few-layer graphene by the exfoliation of graphite in vigorously stirred liquids [30]. Although graphene has been prepared by several other methods, obtaining large quantities used to be very difficult.

Mechanochemistry provided unique phases and microstructures in inorganic materials [31]. High energy milling results in extremely high defect density and forced mixing that destabilize complicated crystal structures and result in simpler metastable crystalline compounds or amorphous materials. Sometimes achieving a certain microstructure, and not the preparation of a new phase, is the desired effect of milling. I fact, mechanical alloying was originally developed to disperse fine oxide particles in an alloy matrix, not to produce new phases. In complex oxides, obtaining the desired defect structure is often the primary objective of very energetic milling [32]. The ability of mechanical processing to make unique materials is 
proven for a broad variety of materials, both inorganic and organic.

\section{Conclusions}

Mechanochemistry benefited substantially from the recent advances in its applications to organic materials. Single-molecule techniques, using AFM or the sonication of a polymer with embedded mechanophores, coupled with appropriate computational techniques, focused fresh attention to the most fundamental form of mechanochemistry: breaking a specific primary bond with mechanical force. On the other hand, organic mechanochemistry also showed that less energetic mechanical grinding, affecting only secondary bonds, can facilitate the preparation of useful compounds with high yield and selectivity. New applications, such as the preparation of cocrystals for pharmaceutical applications, emerged.

The results also call attention to the scarcity of recent similar results on metals and inorganic materials. The mechanism of direct mechanical action, the selection of the bonds that are broken or modified, and the direction dependence of mechanochemical effects are areas that would benefit from new fundamental investigations.

\section{References}

[1] S. James, T. Friščić, guest eds. Chem. Soc. Rev.42, (2013).

[2] Faraday Discussion 170, Mechanochemistry: From Functional Solids to Single Molecules, Montreal 2014.

[3] G. Kaupp, Cryst. Eng. Comm. 11, 388 (2009).

[4] P. Baláž, Mechanochemistry in Nanoscience and Minerals Engineering, Springer-Verlag, Berlin Heidelberg, 2008.

[5] B. Bhushan, C. Kajdas, in B. Bhushan, ed. Fundamentals of Tribology and Bridging the Macro- and Micro/Nanoscales, Kluwer Acad. Pub., the Netherlands 2001, p. 735 .

[6] C. Kajdas, Lubr. Sci. 6, 203 (1994).

[7] S. N. Zhurkov, Intern. J. Fracture Mech. 1, 311 (1965).

[8] L. Takacs, Acta Phys. Pol. A 121, 711 (2012).

[9] L. Takacs, Chem. Soc. Rev. 42, 7649 (2013).

[10] M. C. Lea, Am. J. Sci. 43, 527 (1892).

[11] W. Spring, Bull. Soc. Chim. Fr. 39, 195 and 641 (1883).
[12] W. Spring, Z. anorg. Chem. 6, 176 (1894).

[13] M. C. Lea, Z. anorg. Chem. 7, 50 (1894).

[14] L. Takacs, J. Mater. Sci. 39, 4987 (2004).

[15] E.Z. da Silva, A.J.R. da Silva, A. Fazzio, Phys. Rev. Lett. 87, 256102 (2001).

[16] G. Rubio-Bollinger, S.R. Bahn, N. Agraït, K.W. Jacobsen, S. Vieira, Phys. Rev. Lett. 87, 026101 (2001).

[17] M. K. Beyer, J. Chem. Phys. 112, 7307 (2000).

[18] M. Konôpka, R. Turanský, J. Reichert, H. Fuchs, D. Marx, I. Stich, Phys. Rev. Lett. 100, 115503 (2008).

[19] J. Ribas-Arino, D. Marx, Chem. Rev. 112, 5412 (2012).

[20] A.-S. Duwez, N. Willet (eds.), Molecular Manipulation with Atomic Force Microscopy, CRC Press, Boca Raton 2012.

[21] Z.S. Kean, A.L. Black Ramirez, S.L. Craig, Polymer Chem. 50, 3481 (2012).

[22] J. Morel, S.W.J. den Brook, abstract at the Int. Conf. Deformation Mechanisms, Rheology and Microstructures, Neustadt an der Weinstrasse, Germany 1999.

[23] F.Kh. Urakaev, I.A. Massalimov, Mendeleev Commun. 13, 172 (2003).

[24] S. E. Zhu, F. Li, G. W. Wang, Chem. Soc. Rev. 42, 7535 (2013).

[25] C.S.B. Gomes, P.T. Gomes, M.T. Duarte, J. Organomet. Chem. 760, 101 (2014).

[26] A. Delori, T. Friščić, W. Jones, Cryst. Eng. Comm. 14, 2350 (2012); D. Braga, L. Maini, F. Grepioni, Chem. Soc. Rev. 42, 7638 (2013).

[27] E. A. Losev, E. V. Boldyreva, Cryst. Eng. Comm. 16, 3857 (2014).

[28] K. M. Wiggins, J. N. Brantley, C. W. Bielawski, ACS Macro Lett. 1, 623 (2012).

[29] V. Štrukil, M. D. Igrc, M. Eckert-Maksić, T. Friščić, T. Chem. Eur. J. 18,8464 (2012).

[30] K.R. Paton, E. Varrla, C. Backes, R.J. Smith, U. Khan, A. o'Neill, C. Boland, M. Lotya, O.M. Istrate, P. King, T. Higgins, S. Barwich, P. May, P. Puczkarski, I. Ahmed, M. Moebius, H. Pettersson, E. Long, J. Coelho, S.E. o'Brien, E.K. McGuire, B. Mendoza Sanchez, G.S. Duesberg, N. McEvoy, T.J. Pennycook, C. Downing, A. Crossley, V. Nicolosi, J.N. Coleman, Nature Mater. 13, 624 (2014); J.M. Tour, Nature Mater. 13, 545 (2014).

[31] C. Suryanarayana, Mechanical Alloying and Milling, Marcel Dekker, New York 2004.

[32] A.F. Fuentes, L. Takacs, J. Mater. Sci. 48, 598 (2012). 